\title{
Microscopic Kinetics Pathway of Salt Crystallization in Graphene Nanocapillaries
}

\author{
Lifen Wang $\odot,{ }^{1,2, *, \dagger}$ Ji Chen $\odot,{ }^{3, *}$ Stephen J. Cox $\odot,{ }^{4, *}$ Lei Liu, ${ }^{5,}$ Gabriele C. Sosso $\odot,{ }^{6}$ Ning Li, ${ }^{7,8}$ \\ Peng Gao, ${ }^{7,8}$ Angelos Michaelides $\odot^{4,9,10}$ Enge Wang, ${ }^{1,2,7,11,12}$ and Xuedong Bai $\odot^{1,2,13, \|}$ \\ ${ }^{1}$ Beijing National Laboratory for Condensed Matter Physics, Institute of Physics, Chinese Academy of Sciences, Beijing 100190, China \\ ${ }^{2}$ Songshan Lake Laboratory for Materials Science, Dongguan 523000, China \\ ${ }^{3}$ School of Physics and the Collaborative Innovation Center of Quantum Matters, Peking University, Beijing 100871, China \\ ${ }^{4}$ Yusuf Hamied Department of Chemistry, University of Cambridge, Lensfield Road, Cambridge CB2 1EW, United Kingdom \\ ${ }^{5}$ School of Materials Science and Engineering, Peking University, Beijing 100871, China \\ ${ }^{6}$ Department of Chemistry and Centre for Scientific Computing, University of Warwick, Coventry CV4 7AL, United Kingdom \\ ${ }^{7}$ International Center for Quantum Materials, School of Physics, Peking University, Beijing 100871, China \\ ${ }^{8}$ Electron Microscopy Laboratory, School of Physics, Peking University, Beijing 100871, China \\ ${ }^{9}$ Department of Physics and Astronomy, and Thomas Young Centre, University College London, London WC1E 6BT, United Kingdom \\ ${ }^{10}$ London Centre for Nanotechnology, University College London, London WC1H OAH, United Kingdom \\ ${ }^{11}$ School of Physics, Liaoning University, Shenyang 110036, China \\ ${ }^{12}$ Interdisciplinary Institute of Light-Element Quantum Materials and Research Center for Light-Element Advanced Materials, \\ Peking University, Beijing 100871, China \\ ${ }^{13}$ School of Physical Sciences, University of Chinese Academy of Sciences, Beijing 100190, China
}

(Received 29 November 2020; revised 8 February 2021; accepted 26 February 2021; published 29 March 2021)

\begin{abstract}
The fundamental understanding of crystallization, in terms of microscopic kinetic and thermodynamic details, remains a key challenge in the physical sciences. Here, by using in situ graphene liquid cell transmission electron microscopy, we reveal the atomistic mechanism of $\mathrm{NaCl}$ crystallization from solutions confined within graphene cells. We find that rock salt $\mathrm{NaCl}$ forms with a peculiar hexagonal morphology. We also see the emergence of a transitory graphitelike phase, which may act as an intermediate in a two-step pathway. With the aid of density functional theory calculations, we propose that these observations result from a delicate balance between the substrate-solute interaction and thermodynamics under confinement. Our results highlight the impact of confinement on both the kinetics and thermodynamics of crystallization, offering new insights into heterogeneous crystallization theory and a potential avenue for materials design.
\end{abstract}

DOI: 10.1103/PhysRevLett.126.136001

Understanding and controlling the crystallization of materials from solution is of essential importance in various scientific and technological disciplines, including materials science, biology, geology, and atmospheric science [1,2]. In nature, complex phenomena such as cloud precipitation, biomineralization, and rock formation are associated with crystallization from solution [3], while industrially, solution-based methods offer a relatively simple and lowcost option for mass production [4]. This has motivated a large number of studies aimed at controlling the dynamics of nucleation, for example, the nucleation density, growth rate, and properties of crystals. For instance, by using additives, metals with finer grains, strengthened mechanical properties, and greater resistance to, e.g., salt damage, have been obtained in metallurgy $[5,6]$.

Classical nucleation theory (CNT) gives a largely reasonable description of nucleation and crystallization. However, important questions remain, e.g., whether or not the stable phase nucleates from solution in a single- vs multistep fashion involving intermediate phases [7-12]. While still challenging, in situ graphene liquid cell (GLC) imaging techniques provide a means to elucidate much needed microscopic insights into crystallization mechanisms [13]. Based on the assumption that graphene only interacts weakly with solution, the impact of the GLC on crystallization is often simply interpreted by effects due to reduced dimensionality and nanocapillary pressure, e.g., in studies of confined water [14-16]. However, a delicate balance between the substrate-solute interaction [17-19], the solute-solvent interaction, and thermodynamics under confinement [20] offers a new degree of freedom to modulate the crystallization pathway.

Conventional understanding suggests that $\mathrm{NaCl}$ follows a one-step classical nucleation pathway and grows into its conventional cubic rock salt structure (B1-NaCl) [21,22]. Using atoresolution in situ transmission electron microscopy (TEM), we reveal that, in a GLC, $\mathrm{NaCl}$ unexpectedly crystallizes into hexagonal-shaped crystallites, which predominantly expose their $\{110\}$ facets instead of the conventional $\{100\}$ facets. More surprisingly, a graphiticlike hexagonal $\mathrm{NaCl}$ phase $(h-\mathrm{NaCl})$ [23] appears as an intermediate structure in the crystallization process, hinting 
at a nonclassical nucleation pathway of $\mathrm{NaCl}$ in the GLC. Combined with density functional theory (DFT) calculations and control experiments, we highlight the importance of the interaction between the nascent crystallites and the graphene substrate, which could be considered as a kinetic approach to stabilize the hidden metastable phase and even as a means to effect nonclassical nucleation under confinement more generally.

Our experimental setup, comprising a quasi-twodimensional graphene nanocell, is illustrated in Fig. 1(a). Given the higher electron scattering power of the saturated $\mathrm{NaCl}$ solution, we can identify the solution-encapsulated cells in the suspended TEM grid holes (see Fig. S1 in the Supplemental Material [24]). Figures 1(b), 1(d), and 1(e) show sequential high-resolution TEM images from one crystallization event (Video 1). The corresponding diffractogram is shown in Fig. 1(c), which demonstrates that the nanocrystal has a $\mathrm{B} 1-\mathrm{NaCl}$ structure along the $\{110\}$-zone axis (referred to as " $\{110\}-\mathrm{B} 1-\mathrm{NaCl}$ " hereafter). On-site electron energy loss spectroscopy (EELS) further confirms the $\mathrm{NaCl}$ composition (Fig. S2). The imaged nanocrystals show clear $\{100\}$ and $\{111\}$ facets with $120^{\circ}$ angles, while $\{110\}$ facets also evolve as transient side facets during the growth of this hexagonally shaped nanocrystal; as $\mathrm{NaCl}$ usually crystallizes into a cubic morphology, this observation comes as somewhat of a surprise. Moreover, this observation appears to be a feature of the GLC environment, as control

(a)

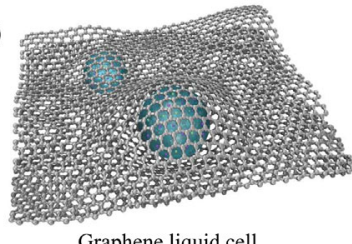

Graphene liquid cell

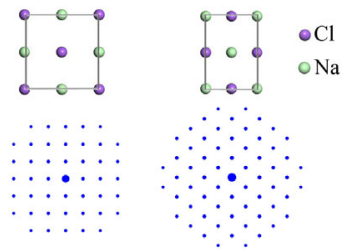

$\{001\}-\mathrm{B} 1-\mathrm{NaCl} \quad\{110\}-\mathrm{B} 1-\mathrm{NaCl}$
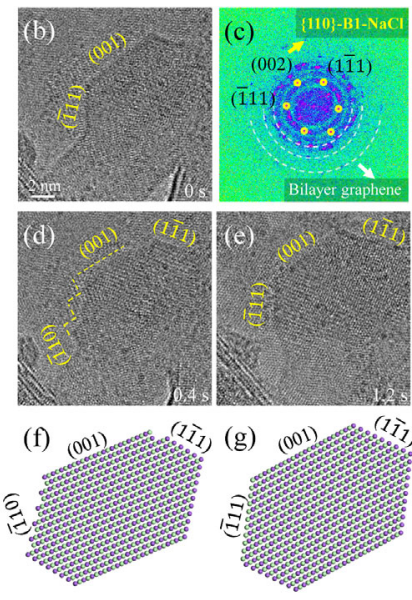

experiments using open $\mathrm{SiN}_{x}$ cells produced B1 crystals with their usual cubic morphology (Fig. S3).

Although the stable B1 phase ultimately forms in our experiments, a different structural phase of $\mathrm{NaCl}$ is occasionally seen to form during the crystallization process. Figures 2(a)-2(d) show high-resolution TEM images along with the corresponding diffractograms. In the diffractograms, the graphene sheets with a rotation angle of $30^{\circ}$ give rise to spots marked by outer white dashed circles (Fig. S4). In addition to these, six spots all with the same vector length from the center can be clearly recognized (yellow circles). These additional spots originate from the $\mathrm{NaCl}$ crystals, which is confirmed using EELS [Fig. 2(e)], and distribute evenly on the circle. Importantly, they differ from the first-order diffraction pattern of a " $\{111\}-\mathrm{B} 1-\mathrm{NaCl}$ " crystal; the $\{111\}$ planes of $\mathrm{B} 1-\mathrm{NaCl}$ are hexagonally close packed so that $\{111\}-\mathrm{B} 1-\mathrm{NaCl}$ also has six equivalent firstorder diffraction spots, but the vector length would be much larger. The sixfold symmetry of spots in reciprocal space thus indicates the formation of a hexagonal crystal structure of $\mathrm{NaCl}(h-\mathrm{NaCl})$, rather than the well-known B1 phase. Moreover, we carefully analyzed the relative angles between the graphene and the $\mathrm{NaCl}$ lattice and find the $\mathrm{NaCl}$ is not in registry with the graphene substrate.

Our assignment of this transient structure as $h-\mathrm{NaCl}$ is corroborated by an analysis of its facet growth dynamics. Figures 2(f)-2(h) show a sequence of TEM images from the longest-lived $h-\mathrm{NaCl}$ crystal. This allows us to track the growth of its facets (Videos 2 and 3). We find two predominant surfaces oriented $30^{\circ}$ with respect to each other, as highlighted by the dashed lines. This is clearly distinct from the $120^{\circ}$ angle seen for $\{110\}-\mathrm{B} 1-\mathrm{NaCl}$, and the $90^{\circ}$ angle expected for a typical B 1 crystal exposing its $\{100\}$ facets. The facet growth shown in Fig. 2 resembles other hexagonal materials such as graphene and hexagonal ice, where competition between the so-called zigzag and armchair edges is likely to evolve. Both the diffractograms and the facet growth dynamics are consistent with characterizing this transient structure as $h-\mathrm{NaCl}$.

The observation of $h-\mathrm{NaCl}$ formation is intriguing and not expected based on conventional understanding of $\mathrm{NaCl}$. To ascertain what role, if any, $h-\mathrm{NaCl}$ plays in the crystallization process, in Fig. 3 we present a sequence of TEM images depicting an entire crystallization event. Insets show the corresponding diffractograms. Initially, only a dark region corresponding to the encapsulated liquid is observed, and no sign of crystallization was seen [Fig. 3(a)]. In the early stages of crystallization, $h-\mathrm{NaCl}$ forms with a well-defined sixfold symmetry [Fig. 3(b)], which after approximately $3 \mathrm{~s}$, begins to shrink [Fig. 3(c)], along with the emergence of B1 crystallites [Fig. 3(d)]. These B1 crystallites subsequently dominate the crystallization process, leading to the formation of a $\{110\}-\mathrm{B} 1-\mathrm{NaCl}$ nanocrystal showing well-marked facets with $120^{\circ}$ angles [Fig. 3(e)]. From these images, we cannot 

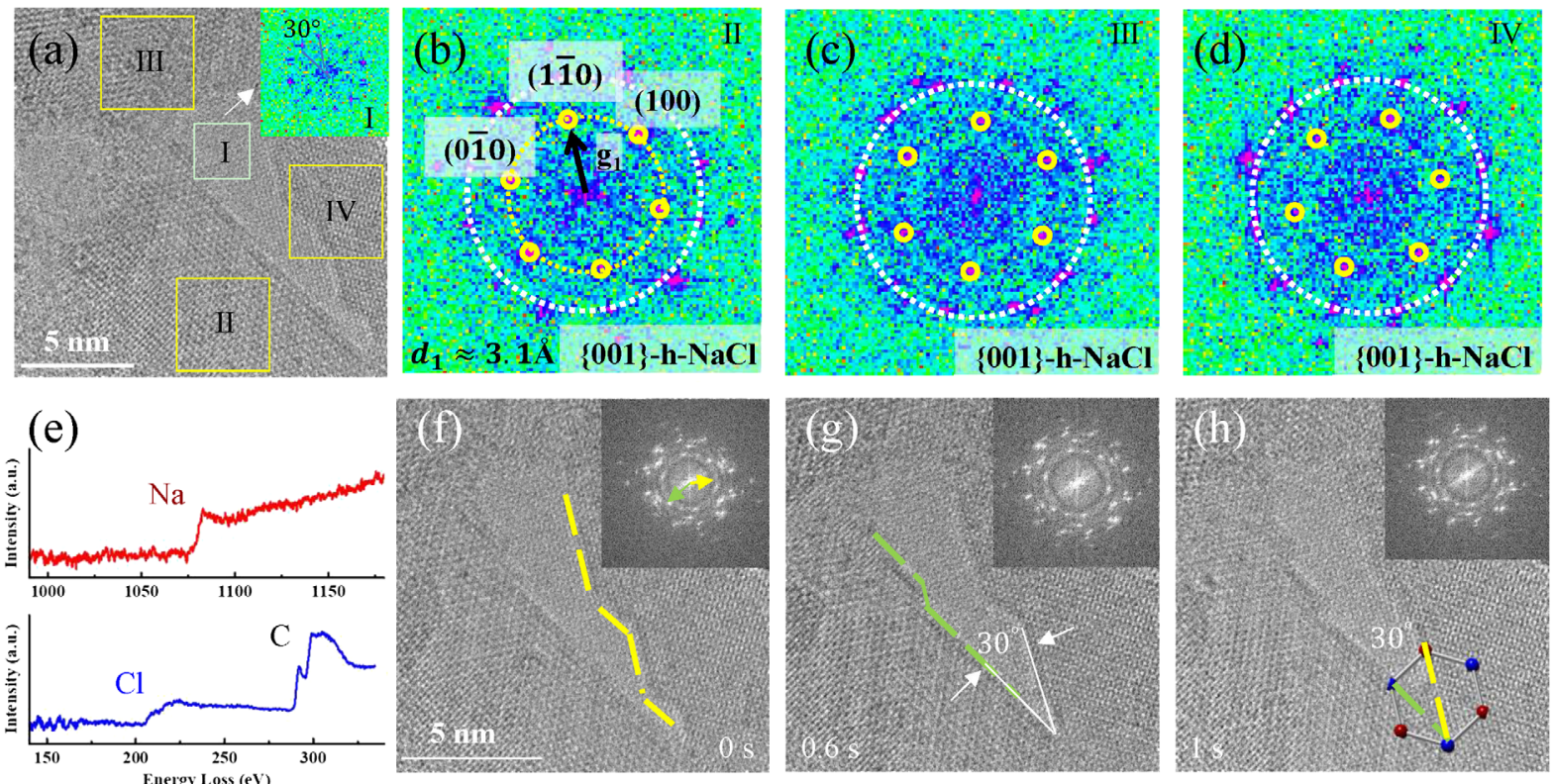

FIG. 2. Formation of the $h$-NaCl phase. (a) TEM image of $\mathrm{NaCl}$ crystals grown in a GLC, showing an in-plane hexagonal lattice. Inset: diffractogram of a graphene-only area with a rotation angle of approximately $30^{\circ}$. (b)-(d) The corresponding diffractograms of selected regions (yellow boxes) in (a), clearly showing sixfold symmetry (inner yellow circles). The rotation angles between $\mathrm{NaCl}$ and graphene (outer white dashed circle) varies. (e) EELS of the pocket area, showing signals only from $\mathrm{C}, \mathrm{Cl}$, and Na. (f)-(h) Sequential TEM images of $h-\mathrm{NaCl}$ crystal growth. The two predominant surfaces have an angle of $30^{\circ}$.

determine if the B1 crystallites have formed via a solid-tosolid transition, or if they have formed independently of $h-\mathrm{NaCl}$. Thus, it remains an open question whether $h-\mathrm{NaCl}$ acts simply as an early - but ultimately unsuccessfulcompetitor of $\mathrm{B} 1-\mathrm{NaCl}$ (similar to recent observations in protein crystallization [41]), or if it acts as an intermediatory phase in a two-step mechanism [42]. The crystallization event shown in Fig. 3 typifies three out of the five events seen in our experiments (Videos 4-7). In the remaining two events, $\{110\}-\mathrm{B} 1-\mathrm{NaCl}$ is seen to form without the detection of $h-\mathrm{NaCl}$, although we cannot preclude, e.g., the prior formation of a thin layer of $h$ - NaCl. On balance, our results lean toward $h-\mathrm{NaCl}$ acting as an unsuccessful competitor to direct $\mathrm{B} 1-\mathrm{NaCl}$ formation,
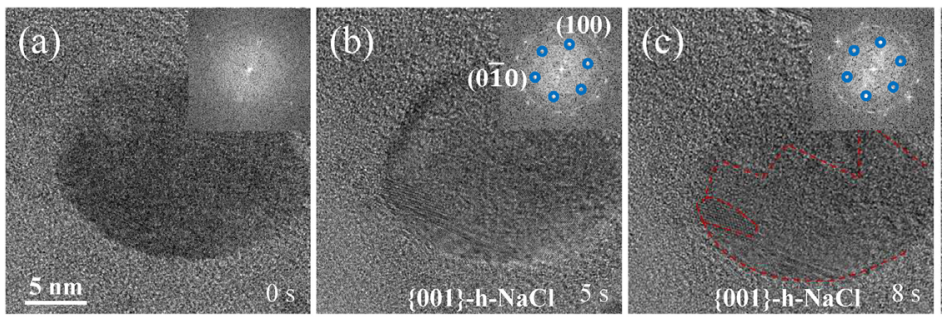

(f)

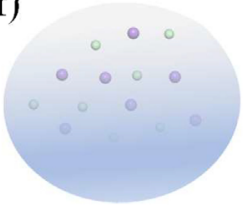

$\mathrm{NaCl}$ solution (g)

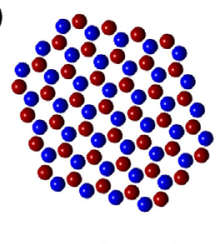

$\{001\}-\mathrm{h}-\mathrm{NaCl}$
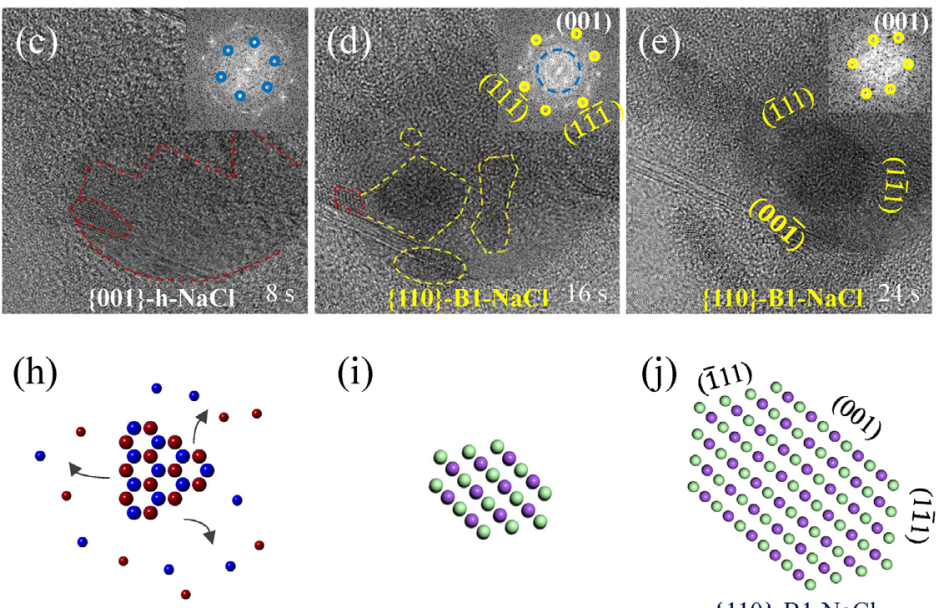

(i)

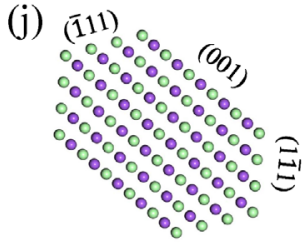

$\{110\}-\mathrm{B} 1-\mathrm{NaCl}$

FIG. 3. Transformation from $h-\mathrm{NaCl}$ to $\{110\}-\mathrm{B} 1-\mathrm{NaCl}$. (a)-(e) Sequential TEM images of a graphene pocket. Dark contrast in the middle of the image indicates the area of the trapped solution. Initially there is no crystal signal from the pocket [(a), inset]. After $5 \mathrm{~s}$ nuclei with the hexagonal structure fill the whole graphene pocket with a uniform lattice (b). Inset of (b) shows spots with sixfold symmetry. In the next stage the hexagonal structure shrinks (c), followed by transformation to $\mathrm{B} 1-\mathrm{NaCl}$ nuclei (d). Dashed red and yellow lines highlight the $h-\mathrm{NaCl}$ and $\mathrm{B} 1-\mathrm{NaCl}$ regions, respectively. Eventually, a large $\{110\}-\mathrm{B} 1-\mathrm{NaCl}$ crystal with a hexagonal shape is observed (e). (f)-(j) Schematics of the corresponding processes in (a)-(e). 
though we cannot definitively rule out a two-step mechanism. In any case, it appears that the crystallization pathway is altered in a GLC environment, even qualitatively, from that in bulk solution.

Previous theoretical studies predict that $h-\mathrm{NaCl}$ is more stable than the B1 phase at large negative pressures, when it is a few layers thin, or when supported by a substrate [43-45]. Previous experimental studies have estimated high pressures (GPa) in GLCs. The fact that $\mathrm{B} 1-\mathrm{NaCl}$ (albeit with an exotic morphology) ultimately forms at the expense of $h-\mathrm{NaCl}$ suggests the former is stable while the latter is metastable. It therefore seems unlikely that pressure underlies the formation of $h-\mathrm{NaCl}$. In order to further understand effects due to solvation and the interactions between the $\mathrm{NaCl}$ crystal and graphene, we have performed DFT calculations for three types of cluster, shown schematically in Figs. 4(a)-4(c): " $\{001\}$-B1" clusters, " $\{110\}-B 1 "$ clusters, and " $h$ clusters." In Fig. 4(d) we present calculated formation energies $e_{f}$ per formula unit for different sized clusters. These calculations have been performed both in vacuum and with an implicit solvent model [46]. We see that the $\{001\}-\mathrm{B} 1$ clusters are significantly more stable than the $\{110\}-B 1$ clusters in vacuum. In solution, however, the calculations with the implicit solvent model $[25,30]$ suggest that the $\{110\}-\mathrm{B} 1$ clusters are marginally (a)

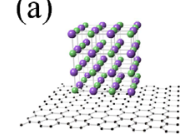

$\{001\} \mathrm{B} \mathrm{i}-\mathrm{NaCl}$

(b)

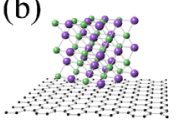

$\{110\} \mathrm{B} 1-\mathrm{NaC}$

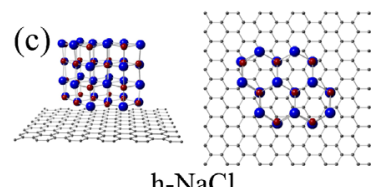

$\mathrm{h}-\mathrm{NaCl}$

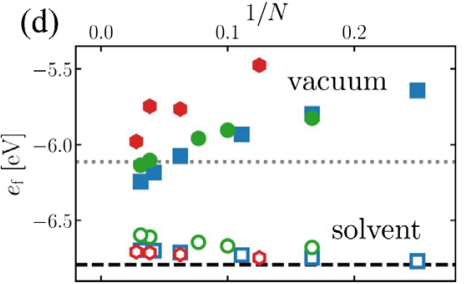

(e)

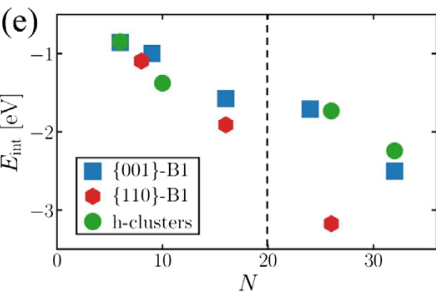

FIG. 4. Energetics of $\mathrm{NaCl}$ clusters. (a)-(c) Schematic representations of $\{001\} \mathrm{B} 1,\{110\}-\mathrm{B} 1$, and $h$ clusters bound to graphene, as indicated at the bottom of each panel. (d) Formation energy per formula unit $\left(e_{f}\right)$ vs $1 / N$. In vacuum, $\{001\}-\mathrm{B} 1$ clusters (squares) are more stable than $\{110\}-\mathrm{B} 1$ clusters (hexagons) for finite $N$. In solvent, the two structures are energetically similar. While the bulk energy of the hexagonal structure (dotted line) is significantly higher than that of the B1 structure (dashed line), the energy difference is far less pronounced for $h$ clusters (circles). (e) Interaction energies with graphene vs $N$. The $\{110\}$-B1 clusters interact much more favorably with graphene than do the $\{001\}$-B1 clusters. The vertical dashed line separates small clusters (all clusters interact with graphene similarly) and large clusters $(\{110\}-B 1$ cluster interacts more strongly with graphene). more stable, which is also reflected in calculations with extended surfaces (Fig. S6). As these results have been obtained with an implicit solvent model we exercise caution, and simply take this as indicative that the difference in surface energies of $\{001\}$ and $\{110\}$ facets is greatly reduced in solution compared to their stark energy difference in vacuum. More importantly, we find that the $\{110\}$-B1 clusters interact much more favorably with graphene than do the $\{001\}$-B1 clusters, as shown in Fig. 4(e). This suggests that the formation of $\{110\}-\mathrm{B} 1-\mathrm{NaCl}$ nanocrystals (Fig. 1) may be driven by a combination solvation effects and a favorable interaction between $\{110\}$ facets and graphene.

In Fig. 4(d) we also see that, while $e_{f}$ for the bulk hexagonal crystal (dotted line) is far higher compared to that of the $\mathrm{B} 1$ structure (dashed line), the $h-\mathrm{NaCl}$ clusters are energetically similar to the B1 clusters. This is the case both in vacuum and with solvent. For very small cluster sizes of $h-\mathrm{NaCl}$, its interaction with graphene [Fig. 4(e)] is comparable to the $\{110\}$-B1 clusters, though it becomes relatively less strong as size increases. During the initial stages of crystallization, we suggest the formation of the $h-\mathrm{NaCl}$ crystal is not disfavored on energetic grounds. As the crystal becomes larger, the preference for the B1 structure increases. These calculations are consistent with our experimental observation that the $\{110\}-\mathrm{B} 1-\mathrm{NaCl}$ nanocrystal ultimately forms.

There is increasing evidence that nucleation occurs nonclassically; notable experimentally observed examples include proteins [7], minerals [47], colloids [48], and polymeric solutions [1]. $\mathrm{NaCl}$, a salt with a simple structure, is believed to follow CNT and shows cubic morphology. Our results suggest elements of nonclassical nucleation extend to $\mathrm{NaCl}$, with the possibility of crystallization via an intermediate metastable crystalline phase: this appears to be distinct from the nonclassical mechanism reported at very high supersaturations [49]. More importantly, our results show that the crystallization pathway in solution can be engineered by the interaction between the crystallites and substrates, i.e., the unexpected transient formation of $h-\mathrm{NaCl}$ as a kinetic stable state in the nanosized capillaries, compared with the conventional cubic structure in the micron-sized open cell. In principle, this approach to achieve a nonclassical nucleation pathway could be readily extended to other systems $[1,2,12]$.

Furthermore, in addition to the revealed crystallization mechanism, our findings clearly show that confinement in a GLC alters crystallization of $\mathrm{NaCl}$ both in terms of morphology and intermediate or transitory metastable phases without involving ultrahigh pressure. This opens up exciting possibilities in nanocrystal design; for example, the morphology controlling ability of the GLC could be useful for catalysis, where the catalytic behavior of a material sensitively depends on exposed facets [50]. Moreover, there has been growing interest in metastable 
crystal structures of functional materials, including the III-V compound semiconductors and transition metal dichalcogenides [51,52]. Future studies may therefore use graphene-confined cells to grow these materials and to discover unknown metastable phases.

We acknowledge G. Gu from University of Tennessee for constructive and fruitful discussions. This work was supported by the Program from Chinese Academy of Sciences (Y8K5261B11, ZDYZ2015-1, XDB30000000, XDB33000000 and XDB07030100), National Natural Science Foundation (11974388, 11974024, 11974001, U1932153, 21872172, 21773303, 51991340 and 51991344), National Key R\&D Program under Grant No. 2019YFA0307801, and Beijing Natural Science Foundation (2192022, Z190011). S. J. C. is supported by a Royal Commission for the Exhibition of 1851 Research Fellowship. L. W. is grateful for the support from the Youth Innovation Promotion Association of CAS (2020009). We thank the TianHe-1A supercomputer, the High Performance Computing Platform of Peking University, and the Platform for Data Driven Computational Materials Discovery of the Songshan Lake Materials Lab for computational resources. We are grateful to the U.K. Materials and Molecular Modelling Hub for computational resources, which is partially funded by EPSRC (EP/P020194/1 and EP/T022213/1).

*L. W., J. C., and S. J. C. contributed equally to this work. †wanglf@iphy.ac.cn;

*jc236@cam.ac.uk

§1_liu@pku.edu.cn

"xdbai@iphy.ac.cn

[1] J. J. De Yoreo, P. U. Gilbert, N. A. Sommerdijk, R. L. Penn, S. Whitelam, D. Joester, H. Zhang, J. D. Rimer, A. Navrotsky, and J. F. Banfield, Science 349, aaa6760 (2015).

[2] G. C. Sosso, J. Chen, S. J. Cox, M. Fitzner, P. Pedevilla, A. Zen, and A. Michaelides, Chem. Rev. 116, 7078 (2016).

[3] D. Gebauer, M. Kellermeier, J. D. Gale, L. Bergström, and H. Cölfen, Chem. Soc. Rev. 43, 2348 (2014).

[4] J. R. Cox, L. A. Ferris, and V. R. Thalladi, Angew. Chem. Int. Ed. 46, 4333 (2007).

[5] W. D. Callister, Jr. and D. G. Rethwisch, Fundamentals of Materials Science and Engineering: An Integrated Approach (John Wiley \& Sons, New York, 2020).

[6] C. Rodriguez-Navarro and L. G. Benning, Elements 9, 203 (2013).

[7] A. Sauter, F. Roosen-Runge, F. Zhang, G. Lotze, R. M. Jacobs, and F. Schreiber, J. Am. Chem. Soc. 137, 1485 (2015).

[8] M. H. Nielsen, S. Aloni, and J. J. De Yoreo, Science 345, 1158 (2014).

[9] J. F. Lutsko, Sci. Adv. 5, eaav7399 (2019).

[10] Y. Peng, F. Wang, Z. Wang, A. M. Alsayed, Z. Zhang, A. G. Yodh, and Y. Han, Nat. Mater. 14, 101 (2015).

[11] Z. Ou, Z. Wang, B. Luo, E. Luijten, and Q. Chen, Nat. Mater. 19, 450 (2020).
[12] V. J. Anderson and H. N. Lekkerkerker, Nature (London) 416, 811 (2002).

[13] J. M. Yuk, J. Park, P. Ercius, K. Kim, D. J. Hellebusch, M. F. Crommie, J. Y. Lee, A. Zettl, and A. P. Alivisatos, Science 336, 61 (2012).

[14] G. Algara-Siller, O. Lehtinen, F. Wang, R. Nair, U. Kaiser, H. Wu, A. Geim, and I. Grigorieva, Nature (London) 519, 443 (2015).

[15] F. Corsetti, P. Matthews, and E. Artacho, Sci. Rep. 6, 18651 (2016).

[16] J. Chen, G. Schusteritsch, C. J. Pickard, C. G. Salzmann, and A. Michaelides, Phys. Rev. Lett. 116, 025501 (2016).

[17] A. Jabbarzadeh and X. Chen, Faraday Discuss. 204, 307 (2017).

[18] A. Jabbarzadeh and B. Halfina, Nanoscale Adv. 1, 4704 (2019).

[19] A. Cacciuto, S. Auer, and D. Frenkel, Nature (London) 428, 404 (2004).

[20] Y. Su, G. Liu, B. Xie, D. Fu, and D. Wang, Acc. Chem. Res. 47, 192 (2014).

[21] N. E. R. Zimmermann, B. Vorselaars, D. Quigley, and B. Peters, J. Am. Chem. Soc. 137, 13352 (2015).

[22] N. Takayuki, S. Masaya, N. Hiroki, H. Koji, and N. Eiichi, J. Am. Chem. Soc. 143, 1763 (2021).

[23] K. A. Tikhomirova, C. Tantardini, E. V. Sukhanova, Z. I. Popov, S. A. Evlashin, M. A. Tarkhov, V. L. Zhdanov, A. A. Dudin, A. R. Oganov, and D. G. Kvashnin, J. Phys. Chem. Lett. 11, 3821 (2020).

[24] See Supplemental Material at http://link.aps.org/ supplemental/10.1103/PhysRevLett.126.136001 for a discussion of the methods used as well as additional experimental and theoretical results, which includes Refs. [24-40].

[25] G. Kresse and J. Furthmüller, Phys. Rev. B 54, 11169 (1996)

[26] S. Plimpton, J. Comput. Phys. 117, 1 (1995).

[27] G. Kresse and D. Joubert, Phys. Rev. B 59, 1758 (1999).

[28] J. Klimeš, D. R. Bowler, and A. Michaelides, J. Phys. Condens. Matter 22, 022201 (2010).

[29] K. Mathew, V. S. C. Kolluru, and R. G. Hennig, VASPsol: Implicit solvation and electrolyte model for densityfunctional theory, GitHub, 2018, https://dx.doi.org/10 .5281/zenodo. 2555053 .

[30] K. Mathew, R. Sundararaman, K. Letchworth-Weaver, T. A. Arias, and R. G. Hennig, J. Chem. Phys. 140, 084106 (2014).

[31] I. S. Joung and T. E. Cheatham, J. Chem. Phys. B 112, 9020 (2008).

[32] J. Klimeš, D. R. Bowler, and A. Michaelides, Phys. Rev. B 83, 195131 (2011).

[33] H. J. C. Berendsen, J. R. Grigera, and T. P. Straatsma, J. Phys. Chem. 91, 6269 (1987).

[34] G. J. Martyna, D. J. Tobias, and M. L. Klein, J. Chem. Phys. 101, 4177 (1994).

[35] M. Parrinello and A. Rahman, J. Appl. Phys. 52, 7182 (1981).

[36] W. Shinoda, M. Shiga, and M. Mikami, Phys. Rev. B 69, 134103 (2004).

[37] M. E. Tuckerman, J. Alejandre, R. López-Rendón, A. L. Jochim, and G. J. Martyna, J. Phys. A 39, 5629 (2006). 
[38] R. W. Hockney and J. W. Eastwood, Computer Simulation Using Particles (Taylor \& Francis, Inc., London, 1988).

[39] J. Kolafa and J. W. Perram, Mol. Simul. 9, 351 (1992).

[40] H. C. Andersen, J. Comput. Phys. 52, 24 (1983).

[41] T. Yamazaki, Y. Kimura, P. G. Vekilov, E. Furukawa, M. Shirai, H. Matsumoto, A. E.S. Van Driessche, and K. Tsukamoto, Proc. Natl. Acad. Sci. U.S.A. 114, 2154 (2017).

[42] D. James, S. Beairsto, C. Hartt, O. Zavalov, I. Saika-Voivod, R. K. Bowles, and P. H. Poole, J. Chem. Phys. 150, 074501 (2019).

[43] A. Kvashnin, E. Pashkin, B. Yakobson, and P. Sorokin, J. Phys. Chem. Lett. 7, 2659 (2016).

[44] Ž. P. Čančarević, J. C. Schoen, and M. Jansen, Chem-Asian J. 3, 561 (2008).

[45] A. G. Kvashnin, P. B. Sorokin, and D. Tománek, J. Phys. Chem. Lett. 5, 4014 (2014).
[46] G. Kresse and J. Furthmüller, Phys. Rev. B 54, 11169 (1996).

[47] F. C. Meldrum and R. P. Sear, Science 322, 1802 (2008).

[48] N. D. Loh, S. Sen, M. Bosman, S. F. Tan, J. Zhong, C. A. Nijhuis, P. Král, P. Matsudaira, and U. Mirsaidov, Nat. Chem. 9, 77 (2017).

[49] H. Jiang, P. G. Debenedetti, and A. Z. Panagiotopoulos, J. Chem. Phys. 150, 124502 (2019).

[50] S. Selcuk and A. Selloni, Nat. Mater. 15, 1107 (2016).

[51] Z. Y. Al Balushi, K. Wang, R. K. Ghosh, R. A. Vilá, S. M. Eichfeld, J. D. Caldwell, X. Qin, Y.-C. Lin, P. A. DeSario, and G. Stone, Nat. Mater. 15, 1166 (2016).

[52] T.-R. T. Han, F. Zhou, C. D. Malliakas, P. M. Duxbury, S. D. Mahanti, M. G. Kanatzidis, and C.-Y. Ruan, Sci. Adv. 1, e1400173 (2015). 